\title{
Nuclear-wave-packet quantum interference in the intense laser dissociation of the $\mathrm{D}_{2}{ }^{+}$molecule
}

\author{
Gábor J. Halász \\ Department of Information Technology, University of Debrecen, H-4010 Debrecen, PO Box 12, Hungary \\ Ágnes Vibók* \\ Department of Theoretical Physics, University of Debrecen, H-40410 Debrecen, PO Box 5, Hungary \\ Nimrod Moiseyev \\ Schulich Faculty of Chemistry, Faculty of Physics, and Minerva Center of Nonlinear Physics in Complex Systems, \\ Technion - Israel Institute of Technology, Haifa 32000, Israel \\ Lorenz S. Cederbaum \\ Theoretische Chemie, Physikalish-Chemisches Institut, Universität Heidelberg H-69120, Germany
}

(Received 13 August 2013; published 11 October 2013)

\begin{abstract}
Recently it has been recognized that electronic conical intersections in molecular systems can be induced by laser light even in diatomics. As is known a direct consequence of these accidental degeneracies is the appearance of nonadiabatic effects which has a strong impact on the nuclear quantum dynamics. Studying the photodissociation process of the $\mathrm{D}_{2}{ }^{+}$molecule, we report here some observable quantum interference phenomena that arise from the topological singularity induced by a strong laser field.
\end{abstract}

DOI: 10.1103/PhysRevA.88.043413 PACS number(s): 34.50.Rk, 33.80.Gj, 34.80.Ht, 33.90.+h

\section{INTRODUCTION}

Conical intersections (CIs) between electronic potential energy surfaces play a key mechanistic role in various basic molecular processes [1-4]. In this case the electronic states are coupled by the nuclear motion, and the energy exchange between the fast electrons and the slow nuclei becomes significant. Owing to the strong nonadiabatic couplings in the close vicinity of these CIs the Born-Oppenheimer adiabatic approximation breaks down. It was pointed out by Longuet-Higgins and Herzberg [5,6] that each real adiabatic electronic state changes sign when transported continuously along a closed path encircling the point of a CI. Mead and Truhlar associated this topological phase effect with the single electronic state problem [7] and Berry generalized the theory [8], hence the name Berry phase. Ensuring that the electronic wave function remains single valued one has to multiply it by a phase factor and, as a consequence of it, this new electronic eigenfunction, instead of being real, becomes complex. The fact that the electronic eigenfunctions are changed has a direct effect on the nuclear dynamics even if it takes place in a single potential energy surface. Therefore, the presence of the Berry phase in a molecular system can be considered as a clear fingerprint of the CI.

CIs in molecules can be formed only if the studied molecular system possesses at least two independent nuclear degrees of freedom. Therefore, for a diatomic molecule that has only one nuclear vibrational coordinate, it is not possible for two electronic states of the same symmetry to become degenerate [unless they belong to a doubly degenerate irreducible representation (e.g., $\pi$ )]. However, this latter statement is true only in a field-free space. The rotation of diatomic molecules exposed to strong laser fields can serve as an additional degree of freedom because the interaction of the induced or permanent dipole moment of the system with the electric field leads to

*Corresponding author: vibok@ phys.unideb.hu an effective torque toward the polarization direction. It was presented in earlier works $[9,10]$ that CIs can be formed both by running or standing lasers waves even in diatomics. In these situations, additional to the nuclear vibration, the rotational motion or the position of the center of mass provide the missing degree of freedom to make possible the formation of a CI. This process is easily understood within the framework of the dressed state representation. Describing the molecule-light interaction in this model, i.e., when the light field is explicitly included into the Hamiltonian, the change of nuclear dynamics due to the light field can be considered as arising from the appearance of a "light-induced conical intersection" (LICI). The energetic and spatial position of these light-induced CIs can be controlled by varying the parameter settings of the laser field. Detailed theoretical investigations demonstrate that these light-induced CIs have a strong impact on the nuclear dynamics [11-15].

In our former works we have pointed out that the appearance of the topological or Berry phase in a molecular system is a clear signature of the CI independently of whether it is a natural or a laser-induced one [11,12]. Applying the line integral technique [16] in the Floquet representation we calculated the Berry phase in diatomics for a LICI situation. One of the clearest observable manifestation of the topological or Berry phase in a molecular system is the appearance of a quantum interference effect $[17,18]$. In order to illustrate this fundamental effect for the present LICI situation, we have chosen as an explicit example the photodissociation of the $\mathrm{D}_{2}{ }^{+}$ molecule, which has been extensively studied for more than two decades [19-24]. In addition, it has also an advantage: Due to its relative simplicity one can study the new light-induced nonadiabatic phenomena separately from other processes.

\section{METHOD}

In our model calculation we will consider the two relevant electronic states of the $\mathrm{D}_{2}{ }^{+}$ion, which are characterized by a 
ground $\left(V_{1}=1 s \sigma_{g}\right)$ and a first excited $\left(V_{2}=2 p \sigma_{u}\right)$ potential energy curve, respectively. For studying the dissociation process the following mechanism will be considered (Fig. 1): Initially the $\mathrm{D}_{2}{ }^{+}$ion is in its ground electronic $\left(1 s \sigma_{g}\right)$ and rotational state combined with one of its vibrational eigenstates. Then, it is excited from this $1 s \sigma_{g}$ state by resonant laser field to the repulsive $2 p \sigma_{u}$ state. As a result of this process the $1 s \sigma_{g}$ and $2 p \sigma_{u}$ electronic states are resonantly coupled by the laser. The nonvanishing dipole matrix elements are responsible for the light-induced electronic transitions. In the space of these two electronic states the following time-dependent Hamiltonian holds for the rovibronic nuclear motions:

$$
H=\left(\begin{array}{cc}
-\frac{1}{2 \mu} \frac{\partial^{2}}{\partial R^{2}}+\frac{L_{\theta \varphi}^{2}}{2 \mu R^{2}} & 0 \\
0 & -\frac{1}{2 \mu} \frac{\partial^{2}}{\partial R^{2}}+\frac{L_{\theta \varphi}^{2}}{2 \mu R^{2}}
\end{array}\right)+\left(\begin{array}{cc}
V_{1}(R) & -\epsilon_{0} f(t) d(R) \cos \theta \cos \omega_{L} t \\
-\epsilon_{0} f(t) d(R) \cos \theta \cos \omega_{L} t & V_{2}(R)
\end{array}\right)
$$

Here $R$ and $(\theta, \varphi)$ are the molecular vibrational and rotational coordinates, respectively, $\mu$ is the reduced mass, and $L_{\theta \varphi}$ denotes the angular momentum operator of the nuclei. Here $\theta$ denotes the angle between the polarization direction and the direction of the transition dipole and thus one of the angles of rotation of the molecule. Although the Hamiltonian depends on the azimuthal angle $\varphi$, in the present situation, as our wave packet always starts from rotational ground state $(J=0)$, there is no $\varphi$ dependance in our results. $V_{1}(R)\left(1 s \sigma_{g}\right)$ and $V_{2}(R)$ $\left(2 p \sigma_{u}\right)$ are the two electronic states coupled by the laser (whose frequency is $\omega_{L}$ and amplitude is $\left.\epsilon_{0}\right), f(t)$ is the envelop function, and $d(R)\left(=-\left\langle\psi_{1}^{e}\left|\sum_{j} r_{j}\right| \psi_{2}^{e}\right\rangle\right)$ is the transition dipole matrix element ( $e=m_{e}=\hbar=1$; atomic units are used throughout the article). The potential energies $V_{1}(R)$ and $V_{2}(R)$ and the transition dipole moment were taken from $[25,26]$. The dressed state representation is very illustrative and helps to understand the essence of the light-induced nonadiabatic

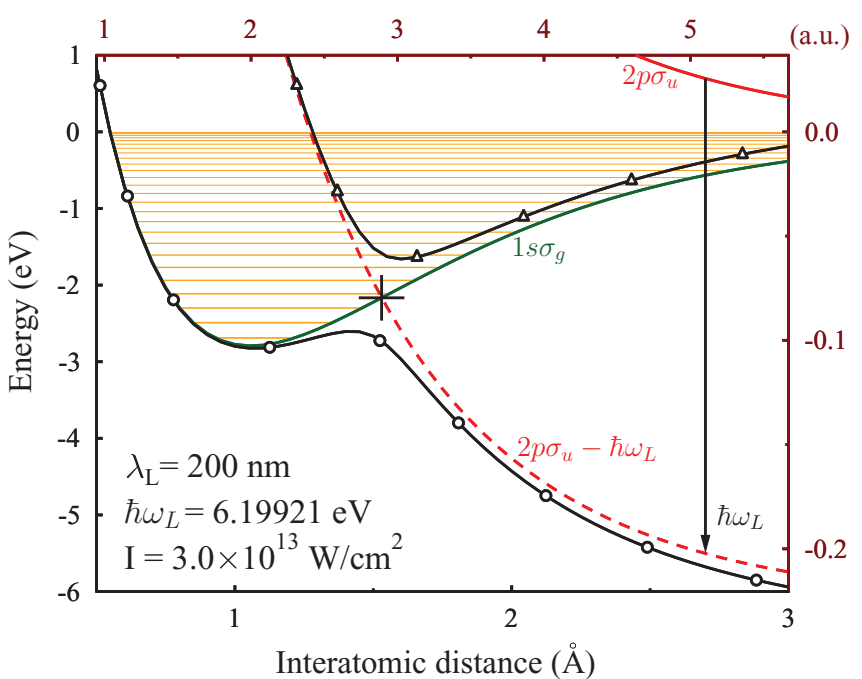

FIG. 1. (Color online) Potential energies of the $\mathrm{D}_{2}{ }^{+}$molecule as a function of interatomic separation. Diabatic energies of the ground $\left(1 s \sigma_{g}\right)$ and the first excited $\left(2 p \sigma_{u}\right)$ states are displayed with solid green and red lines, respectively. The field dressed excited state $\left(2 p \sigma_{u}-\hbar \omega_{L}\right.$; dashed red line) forms a light-induced conical intersection (LICI) with the ground one. For the case of field intensity of $3 \times 10^{13} \mathrm{~W} / \mathrm{cm}^{2}$ a cut of the adiabatic surfaces at $\theta=0$ (parallel with the field) are also shown by solid black lines marked with circles (lower) and triangles (upper). We denoted with a cross the position of the LICI $\left(R_{\mathrm{LICI}}=1.53 \AA=2.891\right.$ a.u. and $\left.E_{\mathrm{LICI}}=-2.16611 \mathrm{eV}\right)$. effects. In this representation the laser light shifts the energy of the $2 p \sigma_{u}$ repulsive excited potential curve by $\hbar \omega_{L}$ and a crossing between the diabatic ground and the diabatic shifted excited potential energy curves is formed. After diagonalizing the potential energy matrix the adiabatic potential surfaces $V_{\text {lower }}$ and $V_{\text {upper }}$ can be obtained (Fig. 1). These two surfaces can cross each other, giving rise to a conical intersection whenever the conditions $\cos \theta=0(\theta=\pi / 2)$ and $V_{1}(R)=V_{2}(R)-\hbar \omega_{L}$ are simultaneously fulfilled.

The characteristic features of the LICI can be changed by varying the frequency and intensity of the laser field. Increasing the frequency, for example, moves the CI to a smaller internuclear distance and to a smaller energetic position. The steepness of the CI cone formed by the adiabatic surfaces, which is related to the strength of the nonadiabatic coupling, can be controlled by the laser intensity. To demonstrate the impact of the LICI on the photodissociation dynamics one has to solve the time-dependent nuclear Schrödinger equation (TDSE) with the Hamiltonian $\hat{H}$ given by Eq. (1). One of the most efficient approaches for solving the time-dependent nuclear Schrödinger equation is the MCTDH (multiconfiguration time-dependent Hartree) method [27-30]. To describe the vibrational degree of freedom we have used FFT-DVR (fast Fourier transformation-discrete variable representation) with $N_{R}$ basis elements distributed within the range from 0.1 to 80 a.u. for the internuclear separation. The rotational degree of freedom was described by Legendre polynomials $\left\{P_{J}(\cos \theta)\right\}_{j=0,1,2, \ldots, N_{\theta}}$. These so called primitive basis sets $(\chi)$ were used to represent the single particle functions $(\phi)$, which in turn were used to represent the wave function

$$
\begin{aligned}
\phi_{j_{q}}^{(q)}(q, t) & =\sum_{l=1}^{N_{q}} c_{j_{q} l}^{(q)}(t) \chi_{l}^{(q)}(q), \quad q=R, \theta, \\
\psi(R, \theta, t) & =\sum_{j_{R}=1}^{n_{R}} \sum_{j_{\theta}=1}^{n_{\theta}} A_{j_{R}, j_{\theta}}(t) \phi_{j_{R}}^{(R)}(R, t) \phi_{j_{\theta}}^{(\theta)}(\theta, t) .
\end{aligned}
$$

In our numerical calculations we have used $N_{R}=2048$ and, depending on the field intensity, $N_{\theta}=6, \ldots, 70$. On both diabatic surfaces and for both degrees of freedom a set of $n_{R}=n_{\theta}=3, \ldots, 25$ single particle functions were used to build up the nuclear wave function of the system. (The actual value of $N_{\theta}$ and $n_{R}=n_{\theta}$ was chosen depending on the peak field intensity $I_{0}$.) All the calculations were correctly converged with these parameters. 
TABLE I. Energies of the studied vibrational levels of the $\mathrm{D}_{2}{ }^{+}$ molecule (in $\mathrm{eV}$ ).

\begin{tabular}{cccccc}
\hline \hline & $v=0$ & $v=1$ & $v=2$ & $v=3$ & $v=4$ \\
\hline$E_{v}$ & -2.6900 & -2.4905 & -2.2985 & -2.1145 & -1.9380 \\
\hline \hline
\end{tabular}

\section{RESULTS AND DISCUSSION}

The solution of Eq. (2) is used to calculate the total dissociation probability and the angular distribution of the photofragments [29]:

$$
P_{\mathrm{diss}}=\int_{0}^{\infty} d t\langle\psi(t)|W| \psi(t)\rangle,
$$

where $-i W$ is the complex absorbing potential (CAP) applied at the last 5 a.u. of the grid related to the vibrational degree of freedom, and

$$
P\left(\theta_{j}\right)=\frac{1}{w_{j}} \int_{0}^{\infty} d t\left\langle\psi(t)\left|W_{\theta_{j}}\right| \psi(t)\right\rangle,
$$

where $-i W_{\theta_{j}}$ is the projection of the CAP to a specific point of the angular grid $\left(j=0, \ldots N_{\theta}\right)$, and $w_{j}$ is the weight related to this grid point according to the applied DVR.

In all of our calculations the initial nuclear wave packet (at $t=0 \mathrm{fs})$ was chosen to be in its rotational ground state $(J=0)$ and in some of its vibrational eigenstate $(v=0,1,2,3,4)$. In this way the angular distribution of the photofragments can provide accurate information about the dissociation of single vibrational levels [20]. Nonaligned (isotropic) molecules and linearly polarized Gaussian laser pulses centered around $t=0 \mathrm{fs}$ were used in the numerical simulations. The center wavelength and the pulse duration at full width of half maximum (FWHM) are $200 \mathrm{~nm}$ and $t_{\text {pulse }}=30$, respectively. We note here that for these values of the laser parameters the $v=0,1,2$ vibrational levels are below the energy of the LICI $\left(E_{\text {LICI }}=-2.1661 \mathrm{eV}\right)$ and the $v=3,4$ states are above it (see Fig. 1 and Table I). We calculated the total dissociation probability $P_{\text {diss }}$ of the photofragments. Results for different intensities are presented in Table II. At low intensities the dissociation probabilities of the low vibrational levels $(v=0,1)$ are small due to the potential barrier. In contrast, the dissociation rate of the $v=3$ and $v=4$ vibrational levels are relatively large for intensity values greater than $1 \times 10^{12} \mathrm{~W} / \mathrm{cm}^{2}$. This

TABLE II. Dissociation probability as a function of the field intensity. (Maximum intensities are given in $\mathrm{W} / \mathrm{cm}^{2}$.)

\begin{tabular}{lcccccc}
\hline \hline$I_{0}$ & $v=0$ & $v=1$ & $v=2$ & $v=3$ & $v=4$ & $v=9$ \\
\hline $1 \times 10^{11}$ & $1.9 \times 10^{-6}$ & $1.7 \times 10^{-4}$ & 0.0037 & 0.0232 & 0.0291 & 0.0120 \\
$3 \times 10^{11}$ & $5.6 \times 10^{-6}$ & $5.1 \times 10^{-4}$ & 0.0113 & 0.0669 & 0.0830 & 0.0353 \\
$1 \times 10^{12}$ & $1.9 \times 10^{-5}$ & 0.0018 & 0.0379 & 0.1948 & 0.2339 & 0.1095 \\
$3 \times 10^{12}$ & $6.6 \times 10^{-5}$ & 0.0060 & 0.1150 & 0.4250 & 0.4715 & 0.2709 \\
$1 \times 10^{13}$ & $3.4 \times 10^{-4}$ & 0.0299 & 0.3612 & 0.7143 & 0.7105 & 0.5263 \\
$3 \times 10^{13}$ & 0.0042 & 0.2081 & 0.7237 & 0.9009 & 0.8757 & 0.6693 \\
$1 \times 10^{14}$ & 0.3051 & 0.7873 & 0.9502 & 0.9868 & 0.9794 & 0.7626 \\
$3 \times 10^{14}$ & 0.9251 & 0.9781 & 0.9962 & 0.9993 & 0.9956 & 0.8769 \\
\hline \hline
\end{tabular}

feature can easily be explained borrowing the light-induced potential (LIP) picture [19]. The molecules are exposed to small and intermediate light intensities and follow the diabatic dissociation path, while for high enough intensities they rather tend to follow the adiabatic path. For small light intensities the dissociation behaves linearly with the intensity, whereas at higher intensities up to $10^{14} \mathrm{~W} / \mathrm{cm}^{2}$ this quantity is strongly nonlinear. In the present case the $v=0,1,2$ levels lie in the lower adiabatic potential. As for the other states, they are in the gap between the two adiabatic potential curves. The number of vibrational states in the gap region depends on the value of the intensity. Increasing the intensity, the gap between the two adiabatic potential curves is increasing. As a consequence, the so-called bond softening mechanism occurs for states $v=0,1,2$ (see in Table II for $10^{14} \mathrm{~W} / \mathrm{cm}^{2}$ ), while for higher vibrational states, which are not in the gap region, the well known trapping effect or bond hardening emerges (see the last column of Table II). For those vibrational states that are in the gap region the transition probability is relatively large. Neither bond hardening nor bond softening occurs. For high enough intensities almost all molecules from these states dissociate following the nonadiabatic path. In our case the $v=3$ and $v=4$ vibrational states are in the gap region and provide almost $100 \%$ dissociation rate at $10^{14} \mathrm{~W} / \mathrm{cm}^{2}$. As a next step we calculate the fragment angular distribution. The obtained results are summarized in Fig. 2. Regarding the structures of these curves the following observation can be made: Curves are very smooth for $1 \times 10^{12} \mathrm{~W} / \mathrm{cm}^{2}$, but for large enough intensities $\left(1 \times 10^{14} \mathrm{~W} / \mathrm{cm}^{2}\right)$ some distinct modulations appear on them. Similar results were obtained in a very recent paper of Fischer and co-workers [24]. They have studied the photodissociation of the $\mathrm{H}_{2}{ }^{+}$molecule solving the TDSE including the rotational dynamics. They considered vibrationally and rotationally excited initial wave packet $(v=4, J=2)$, and the results obtained are presented in Fig. 3(c) of [24]. Their conditions are very similar to ours, but since the applied wavelength is $\operatorname{larger}(\lambda=266 \mathrm{~nm})$ the position of the LICI is shifted to the right. Consequently, the $v=3$ level is still below the energy of the CI but the $v=4$ state already lies above it. After carefully checking the laser parameters they used, it seems that the $v=4$ level of $\mathrm{H}_{2}{ }^{+}$sits in the gap between the two light-induced adiabatic potentials. The $v=4$ level plays the same role in their study as $v=3$ in our case. Both sit on the gap region. Correspondingly, Fig. 3(c) in [24] is very similar to ours (Fig. 2, $I=1 \times 10^{14}$ $\mathrm{W} / \mathrm{cm}^{2}$, curve for $v=3$ ), despite the fact that two different theoretical methods were used for the solution of the TDSE. The distinct modulations and the abrupt decreases for angles $\theta \geqslant 60^{\circ}$ appear in both pictures. There is only one difference between the two initial wave packets. Our wave packet is in its rotationally ground state $(J=0)$, whereas theirs is rotationally excited $(J=2)$. This, however does not mean essential difference, because the LICI introduces an intense nonadiabatic coupling via mixing the rotational and vibrational motions on both electronic surfaces and, therefore, even if one starts with $J=0$, afterwards it would have $J$ values up to 30 or 40. To understand more deeply the modulations on the curves, we have performed the analysis of the nuclear density function $|\psi(R, \theta, t)|^{2}\left[=\left|\psi^{1 s \sigma_{g}}(R, \theta, t)\right|^{2}+\left|\psi^{2 p \sigma_{u}}(R, \theta, t)\right|^{2}\right]$. Obtained results are illustrated in Fig. 3 with snapshots from the structure 

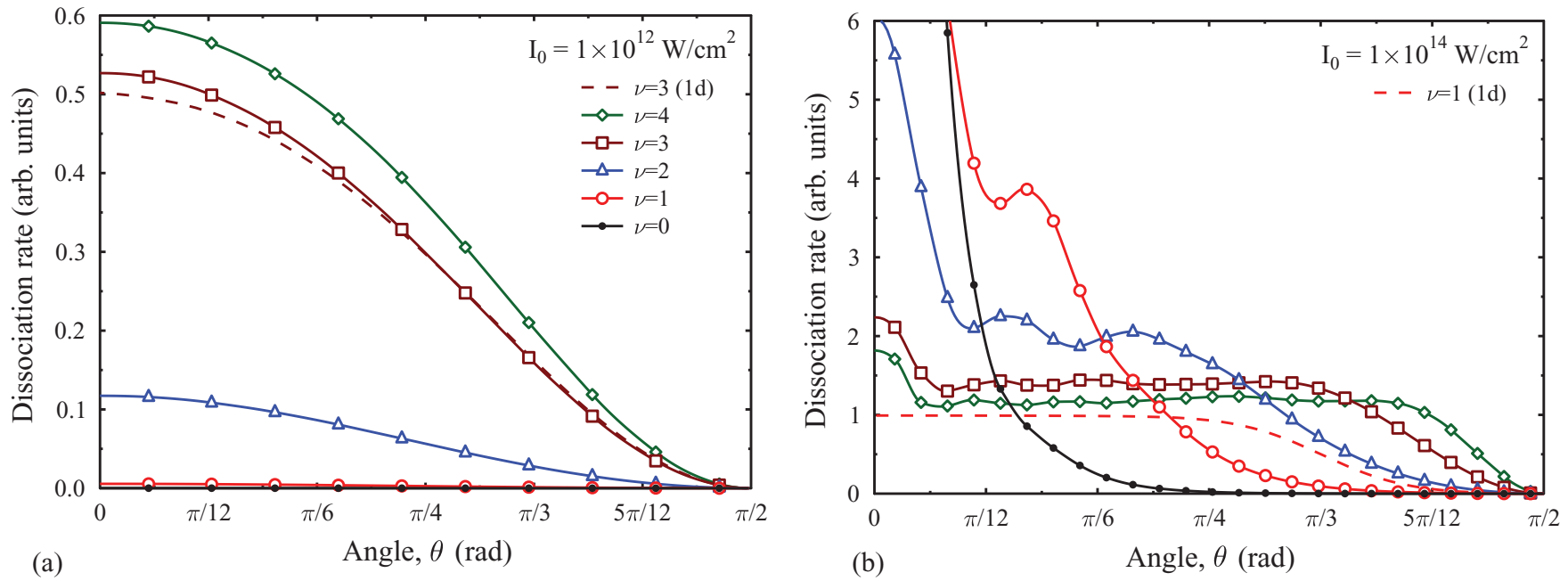

FIG. 2. (Color online) Fragment angular distributions of the dissociating $\mathrm{D}_{2}{ }^{+}$molecule for two different intensities $\left(1 \times 10^{12}\right.$ and $1 \times 10^{14}$ $\left.\mathrm{W} / \mathrm{cm}^{2}\right)$ and five different vibrational states $(v=0,1,2,3,4)$. One-dimensional (1D) calculations are presented for $v=3$ at $1 \times 10^{12}$ and $v=1$ at $1 \times 10^{14} \mathrm{~W} / \mathrm{cm}^{2}$ intensities.

of the nuclear wave packet density $|\psi(R, \theta, t)|^{2}$ at different times. It is seen that the nuclear wave packet passes through the dissociation region, but owing to the strong nonadiabatic topological effect of the LICI, it is unable to move unhindered. It splits up in front of the LICI region, bypasses both sides of the LICI, and then its two components exhibit a complex motion. On the one hand, they move horizontally from left to the right, which would represent the pure dissociation, but on the other hand, they also move in a vertical direction from top to bottom and vice versa. Due to the rotation induced by the external electric field rotational nodes being formed roughly after the maxima of the intensity is reached $(t=0 \mathrm{fs})$.
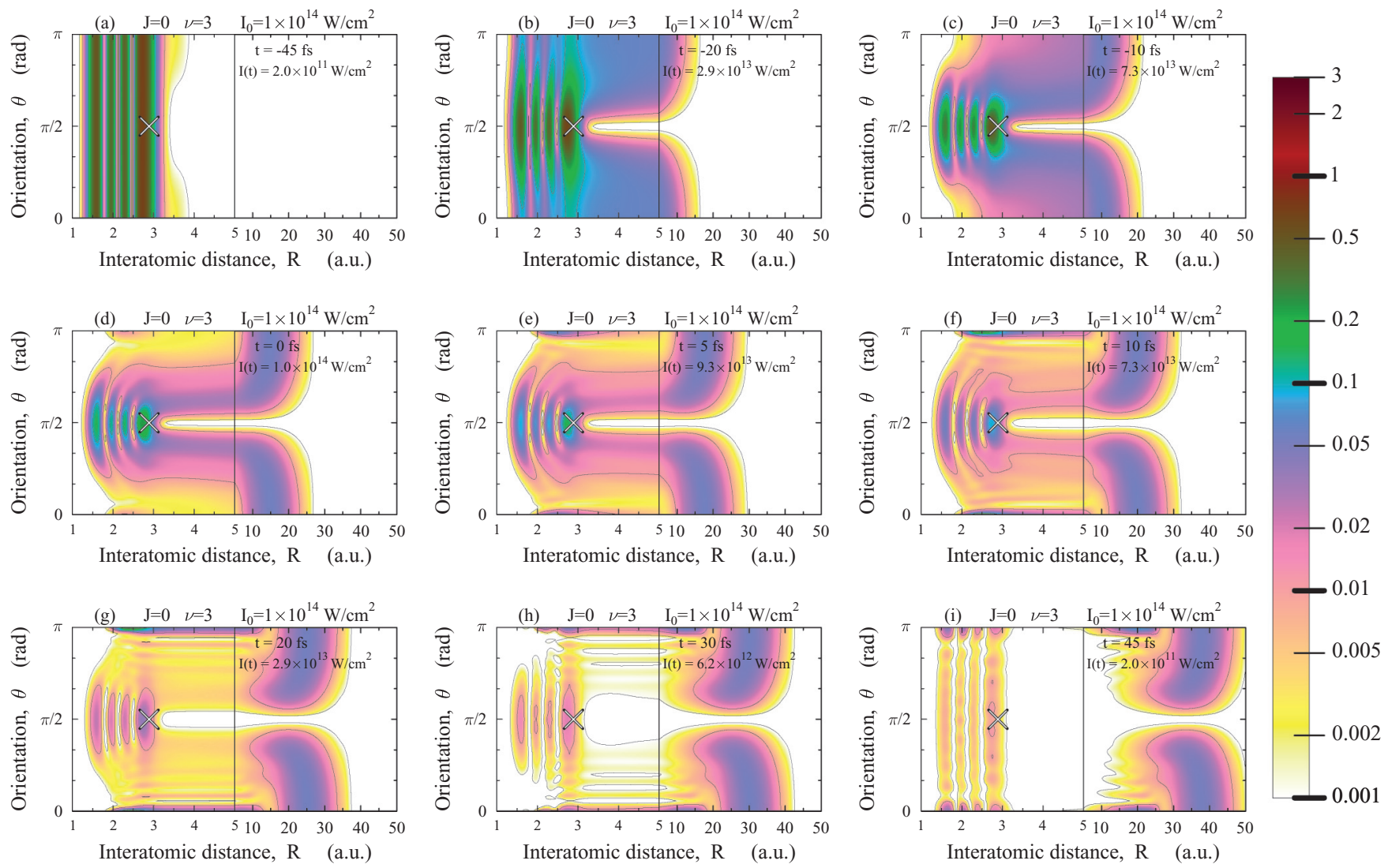

FIG. 3. (Color online) Snapshots from the real-time evolution of the interference appearing in the angular distribution of the dissociated particles. $I=1 \times 10^{14} \mathrm{~W} / \mathrm{cm}^{2}$ intensity and $v=3$ vibrational state are applied. The cross denotes the position of the LICI. 

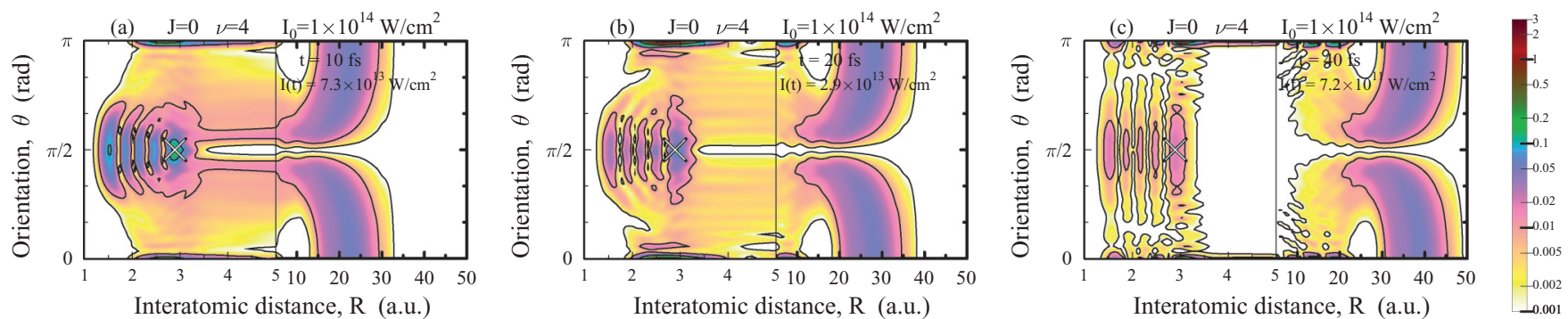

FIG. 4. (Color online) Snapshots from the real-time evolution of the nuclear wave packet density. $I=1 \times 10^{14} \mathrm{~W} / \mathrm{cm}^{2}$ intensity and $v=4$ vibrational state are applied. The cross denotes the position of the LICI.

It means that at given directions the wave function disappears from the bounded region of the configuration space. As a consequence of it, the dissociation in these directions will also be reduced. But in contrast, above and below these nodes the value of the wave packet density is significantly different from zero. So-called quantum interference effects appear, maxima turn into minima, and vice versa. These quantum interference effects are the sources of the bumpy structure appearing in the angular distribution of the photofragments [see Fig. 2(b)]. We note here that for symmetry reasons while the one photon processes are dominant in the laser-matter interaction, the nuclear wave packet should vanish at $\theta=\pi / 2$ in the dissociation region.

It is worth mentioning that even at high intensities the sign of the interference in the angular distribution does not always appear. For example, for the level of $v=0$ due to bond softening the dissociation takes place near parallel to the polarization of the field, and the dissociation probability changes so rapidly as the function of the angle that the small modulation caused by the rotational nodes is not visible in the angular distribution (Fig. $2, I=1 \times 10^{14} \mathrm{~W} / \mathrm{cm}^{2}$ ). On the other hand, for $v=4$ we obtain very rich interference patterns in the nuclear wave packet density (Fig. 4) but owing to integration, the fingerprints of this interference disappears from the angular distribution (Fig. 2).

We note here that the situation is quite different for small and moderate intensity values as such $I=1 \times 10^{12} \mathrm{~W} / \mathrm{cm}^{2}$. For any given vibrational state, there is no interference pattern

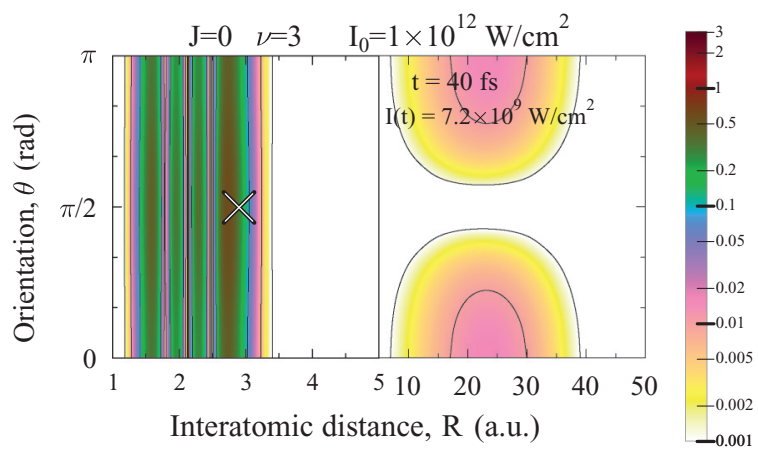

FIG. 5. (Color online) Snapshot from the real-time evolution of the nuclear wave packet density. $I=1 \times 10^{12} \mathrm{~W} / \mathrm{cm}^{2}$ intensity and $v=3$ vibrational state are applied. The cross denotes the position of the LICI. appeared in the nuclear density function (see, e.g., Fig. 5 for $v=3$ ). The applied field is not strong enough to build up notable rotational nodes.

In summary, the results obtained clearly confirm that additional structures or modulations appear in the angular distributions strongly related to the interference properties of the nuclear wave packet.

Additionally, one-dimensional calculations (1D) for the angular distribution have also been performed. We froze the rotational degree of freedom and accordingly the LICI was not considered. With such conditions, the molecule's initial orientation was not changing during the dissociation process and the "effective field strength" was the projection of the real field to the axis of the molecule: $\epsilon_{0}^{\text {eff }}=\epsilon_{0} \cos \theta\left(I_{0}^{\text {eff }}=\right.$ $\left.I_{0} \cos ^{2} \theta\right)$. In such cases we have never obtained modulations on the angular distribution curves similar to the ones observed in the full calculations (Fig. 2). (The scale on Fig. 2 was chosen so that the dissociation rate of 1 represents the total dissociation in a given direction. Larger values in the full calculation mean that some parts of the dissociating particles were rotated by the field to this direction from some different initial ones.)

\section{CONCLUSIONS}

The main issue discussed in this work is related to the rotation. By means of two-dimensional quantum dynamical calculations, we have demonstrated that the additional rotational degree of freedom in the description of the $\mathrm{D}_{2}{ }^{+}$ photodissociation promises a wealth of observable quantum phenomena. After intense laser-matter interaction, a measurable quantum interference effect occurs in the molecular system due to the very strong nonadiabatic coupling of the electronic, rotational, and vibrational motions.

\section{ACKNOWLEDGMENTS}

The authors acknowledge financial support by the Deutsche Forschungsgemeinschaft (Project ID CE10/50-1). Á.V. acknowledges the computational resources provided by the John-von-Neumann Institute, Research Centre Juelich (Project ID ehu01), the OTKA Grant No. NN103251. This research was partly supported by the European Union and the State of Hungary, co-financed by the European Social Fund in the framework of TÁMOP 4.2.4. A/2-11-1-2012-0001 'National Excellence Program'. 
[1] H. Köppel, W. Domcke, and L. S. Cederbaum, Adv. Chem. Phys. 57, 59 (1984).

[2] G. A. Worth and L. S. Cederbaum, Annu. Rev. Phys. Chem. 55, 127 (2004)

[3] W. Domcke, D. R. Yarkony, and H. Köppel, Conical Intersections: Electronic Structure, Dynamics and Spectroscopy (World Scientific, Singapore, 2004).

[4] M. Baer, Beyond Born Oppenheimer: Electronic Non-Adiabatic Coupling Terms and Conical Intersections (Wiley, New York, 2006).

[5] G. Herzberg and H. C. Longuet-Higgins, Discuss. Faraday Soc. 35, 77 (1963).

[6] H. C. Longuet-Higgins, Proc. R. Soc. London Ser. A 344, 147 (1975).

[7] C. A. Mead and D. G. Truhlar, J. Chem. Phys. 70, 2284 (1979).

[8] M. V. Berry, Proc. R. Soc. London Ser. A 392, 45 (1984).

[9] N. Moiseyev, M. Sindelka, and L. S. Cederbaum, J. Phys. B 41, 221001 (2008).

[10] M. Sindelka, N. Moiseyev, and L. C. Cederbaum, J. Phys. B 44, 045603 (2011).

[11] G. J. Halász, Á. Vibók, M. Sindelka, N. Moiseyev, and L. S. Cederbaum, J. Phys. B 44, 175102 (2011).

[12] G. J. Halász, M. Sindelka, N. Moiseyev, L. S. Cederbaum, and Á. Vibók, J. Phys. Chem. A 116, 2636 (2012).

[13] G. J. Halász, Á. Vibók, M. Sindelka, L. S. Cederbaum, and N. Moiseyev, Chem. Phys. 399, 146 (2012).

[14] G. J. Halász, Á. Vibók, N. Moiseyev, and L. S. Cederbaum, J. Phys. B 45, 135101 (2012).

[15] G. J. Halász, Á. Vibók, H.-D. Meyer, and L. S. Cederbaum, J. Phys. Chem. A 117, 8528 (2013).
[16] M. Baer, Chem. Phys. 259, 123 (2000).

[17] R. Y. Chiao and Y. S. Wu, Phys. Rev. Lett. 57, 933 (1986).

[18] A. Tomita and R. Y. Chiao, Phys. Rev. Lett. 57, 937 (1986).

[19] A. D. Bandrauk, E. Aubanel, and J. M. Gauthier, Molecules in Laser Fields (Marcel Dekker, New York, 1994).

[20] K. Sandig, H. Figger, and T. W. Hansch, Phys. Rev. Lett. 85, 4876 (2000)

[21] V. N. Serov, A. Keller, O. Atabek, and N. Billy, Phys. Rev. A 68, 053401 (2003).

[22] F. Anis, T. Cackowski, and B. D. Esry, J. Phys. B 42, 091001 (2003).

[23] F. Anis and B. D. Esry, Phys. Rev. A 77, 033416 (2008).

[24] M. Fischer, U. Lorenz, B. Schmidt, and R. Schmidt, Phys. Rev. A 84, 033422 (2011).

[25] F. V. Bunkin and I. I. Tugov, Phys. Rev. A 8, 601 (1973).

[26] S. I. Chu, C. Laughlin, and K. Datta, Chem. Phys. Lett. 98, 476 (1983).

[27] H. D. Meyer, U. Manthe, and L. S. Cederbaum, Chem. Phys. Lett. 165, 73 (1990).

[28] U. Manthe, H. D. Meyer, and L. S. Cederbaum, J. Chem. Phys. 97, 3199 (1992).

[29] M. H. Beck, A. Jackle, G. A. Worth, and H. D. Meyer, Phys. Rep. 324, 1 (2000); G. A. Worth et al., The MCTDH package, version 8.2 (2000), version 8.3 (2002), version 8.4 (2007), University of Heidelberg, Germany; http://mctdh.uni-hd.de/

[30] H. D. Meyer, F. Gatti, and G. A. Worth, Multidimensional Quantum Dynamics: MCTDH Theory and Applications (Wiley-VCH, Weinheim, 2009). 